\title{
Illusory 3-D rotation induced by dynamic image shading
}

\author{
CORRADO CAUDEK \\ Università degli Studi di Trieste, Trieste, Italy \\ FULVIO DOMINI \\ Brown University, Providence, Rhode Island \\ and \\ MASSIMILIANO DI LUCA \\ Università degli Studi di Trieste, Trieste, Italy
}

\begin{abstract}
Observers' perceptions of the three-dimensional structure of smoothly curved surfaces defined by patterns of image shading were investigated under varying conditions of illumination. In five experiments, observers judged the global orientation and the motion of the simulated surfaces from both static and dynamic patterns of image shading. We found that perceptual performance was more accurate with static than with dynamic displays. Dynamic displays evoked systematic biases in perceptual performance when the surface and the illumination source were simulated as rotating in opposite directions. In these conditions, the surface was incorrectly perceived as rotating in the same direction as the illumination source. Conversely, the orientation of the simulated surfaces was perceived correctly when the frames making up the apparent-motion sequences of the dynamic displays were presented as static images. In Experiment 6, moreover, the results obtained with the computer-generated displays were replicated with solid objects.
\end{abstract}

Image shading (smooth variation in image luminance) is an effective two-dimensionalcue for evoking a compelling impression of three-dimensional (3-D) shape. Several psychophysical investigationshave illustrated this point (e.g., Erens, Kappers, \& Koenderink, 1993a, 1993b; Johnston \& Passmore, 1994a, 1994b; Mingolla \& Todd, 1986; Pollick, Watanabe, \& Kawato, 1996; Ramachandran, 1988a, 1988b; Reichel, Todd, \& Yilmaz, 1995; Todd \& Mingolla, 1983; Todd \& Reichel, 1989). Shading information, however, is inherently ambiguous (for a discussion, see D'Zmura, 1991; Todd \& Reichel, 1989). The luminance at every point of the image, in fact, depends on the illuminant direction, the surface's orientation, and the surface's reflectance properties. The same pattern of shading, therefore, can be generated by different combinations of these variables. In principle, however, the knowledge of the illuminant direction is sufficient to disambiguate image shading. Computer vision algorithms relying on the estimate of the illuminant direction, in fact, have been devised for reconstructing surface structure from image shading (e.g., Horn, 1975, 1977; Pentland, 1984, 1989; for a review of the computational analysis of shape from shading, see Horn \& Brooks, 1989).

Even if the knowledge of the illuminant direction is, in principle, sufficient to disambiguate shape from shading,

Correspondence concerning this article should be addressed to C. Caudek, Dipartimento di Psicologia, Università degli Studi di Trieste, Via S. Anastasio 12,34100Trieste, Italy (e-mail: caudek@univ.trieste. it). it has also been demonstrated that this knowledge is not necessary for finding a solution to the shape-from-shading problem (Koenderink \& van Doorn, 1980, 1993). Some 3-D properties, in fact, can be directly recovered from a static pattern of image shading, with no mediating steps representing the conditions in the world that could have produced the observed luminance distribution. Since the topological properties (but not the Euclidean properties) could be derived in this fashion, this alternative approach raises the question of establishing which kind of geometric description is adequate for the 3-D shape actually recovered by the human visual system from image shading. Several psychophysical investigations have addressed this question (e.g., Koenderink, Kappers, Todd, Norman, \& Phillips, 1996; Koenderink \& Lappin, 1996; Koenderink, van Doorn, Christou, \& Lappin, 1996a, 1996b; Koenderink, van Doorn, \& Kappers, 1992; Koenderink, van Doorn, Kappers, \& Todd, 1997). A sketchy way to differentiate the two formal approaches mentioned above is to say that the first one (e.g., Pentland, 1984) performs a local analysis, whereas the second one (e.g., Koenderink \& van Doorn, 1980) is more global in nature (for a discussion on the local/global status of shading information, see Mingolla \& Todd, 1986).

The perception of image shading generated by varying the illuminant direction provides an interesting problem for vision research. In this paper, we examine perceptual performance when observers were shown static or dynamic patterns of image shading. Given that several reports indicate that the manipulation of the illuminant direction induces 
systematic distortions in the perception of 3-D shape from static patterns of image shading (e.g., Christou \& Koenderink, 1997; Todd, Koenderink, van Doorn, \& Kappers, 1996), we asked whether perceptual performance can improve when observers are shown a dynamic shading display.

It may be that the illuminant direction can be recovered with better accuracy from dynamic displays and, consequently, the continuous variations of the illuminant direction over time might not induce the systematic distortions reported with static displays. Conversely, the transformations of the luminance gradients caused by the variations of the illuminant direction may be better accounted for in static images than when they occur over time within a dynamic image. In a natural environment, in fact, the illuminant direction remains roughly constant over short periods of time. As a consequence, the perceptual system may have evolved by embodying the assumption that changes of luminance intensities over time are more likely to be due to changes of the surfaces' orientation relative to a stationary illumination source, rather than to changes of the illuminant direction. On these grounds, therefore, one could argue also that the perception of dynamic shading may be systematically distorted, at least for dynamic shading produced by a changing illuminant direction.

Alternatively, perceived shape from shading may not involve the derivation of the illuminant direction in order to reduce the number of unknowns in the ill-posed relationship between shading and surface orientation; rather, 3-D shape may be recovered directly. The problem remains, however, as to whether the perceptual analysis of static shading carries over to the case of dynamic displays (so that the final percept combines the processing of each image of the motion sequence treated as a static shading pattern) or the motion signals present in dynamic shading require an inherently different kind of processing dependent on the specific dynamic properties of the displays. The first hypothesis implies that, with a dynamic shading pattern, performance could remain the same as with a static pattern or it could improve (since more samples of the target object are provided). The second hypothesis, conversely, leaves open the possibility that the perception of dynamic shading may exhibit different properties than does the perception of static shading patterns.

The intent of the present investigation was to address these questions by investigating, in both static and dynamic images, the perception of shading patterns generated by a varying illuminant direction.

\section{Influence of the Illuminant Direction on Perceived Shape From Shading}

The knowledge of illuminant direction encodes, in principle, useful information for recovering 3-D shape from image shading. The changes in luminance at every point of an image, in fact, depend primarily on the relative orientation between the object's surface (defined by the orientation of the plane tangent to the surface) and the illuminant. A small change in the surface's orientation parallel to the illuminant direction produces a large change in the intensity of light reflected by the surface; conversely, if the surface's orientation is orthogonal to the illuminant direction, the same luminance change would require a very large change of the orientation of the surface. By knowing illuminant direction, then, it is possible in principle to map changes in image intensity onto changes in surface orientation (see Pentland, 1982). Psychophysical evidence suggesting that the knowledge of the illuminant direction influences perceptual performance has also been provided. The perceived convexity/ concavity of a surface region, for example, is influenced by the observer's judgment of the illuminant direction (Ramachandran, 1988a, 1988b).

In some circumstances, human observers are capable of estimating the illuminant direction with good accuracy. By using digitized natural images, Pentland (1982) reported that perceptual estimates of illuminant direction had an error of not more than $10^{\circ}$. Furthermore, Todd and Mingolla (1983) reported that judgments of the direction of illumination can be quite accurate also for artificial displays consisting of simulated shiny and dull untextured cylindrical surfaces. By using randomly shaped shaded objects, Todd and Norman (1999) found discrimination thresholds for illuminant direction as small as $4^{\circ}$.

Even though, in some circumstances, the illuminant direction is perceived with good accuracy, this does not mean that perceived shape from shading is accurate. Particularly relevant for the present investigation are reports indicating that systematic distortions in the perceived 3-D shape are produced by changing the direction of illumination in static patterns of image shading. Christou and Koenderink (1997), for example, simulated simple 3-D shapes consisting of spheres or ellipsoids. Observers were asked to adjust attitude probes in different image locations as the position of the illumination source was varied across stimulus displays. Christou and Koenderink reported small but systematic violations of shape constancy, described as a regression to image luminance: "When the light source was from the left there was a slight bulge of reconstructed shape on the left; when the illumination was from the right, the reconstructed shape bulged slightly to the right" (p. 1449).

By using the same procedure, similar results were reported by Todd et al. (1996) for the perception of a male and a female torso with either frontal or oblique illumination. A first effect of the illuminant direction was that oblique illumination produced a greater amount of pictorial relief than did frontal illumination. A second effect of the illuminant direction was that changing the illuminant direction changed the perceived structure of the torsos in a piecewise manner (the perception of local relief changed as illumination direction was varied).

By using laser-scanned face models, Troje and Siebeck (1998) reported an effect of illuminant direction on the global perceived orientation of a human face. They found that variation of the illuminant direction induced an apparent orientation shift of the face in the direction opposite to the illumination change. The magnitude of the apparent orientation shift was smaller for frontal views of the face and when the outline of the face was fully visible. 
All these investigations show that the perception of static image shading may be systematically distorted by varying illuminant direction (but see also Johnston \& Passmore [1994a, 1994b], Reichel \& Todd [1990], and Todd \& Reichel [1989], where changing the direction of illumination had no effect on performance). The intent of the present study was to extend these findings by comparing the perceptual effects deriving from the variation of the illuminant direction in static patterns of image shading with the perceptual effects deriving from the continuous variation of the illuminant direction in dynamic patterns of image shading. In particular, we compared perceptual performance when static images were used and when the same images were presented within an apparent-motion sequence simulating a continuous change of orientation over time between a 3-D shape and an illumination source.

The main finding of the present research is that the dynamic patterns of image shading can be systematically misperceived even if the images used for the apparent-motion sequence of the dynamic displays are perceived veridically when they are shown in isolation (as static displays). The misperceptions described in the present experiments occur for dynamic patterns of image shading representing surfaces rotating under a continuously changing illuminant direction. In these circumstances, a surface rotating about a vertical axis in the clockwise direction (for example) may give rise to the perception of a surface that is rotating in the clockwise or in the counterclockwise direction, depending on the direction of rotation of the illumination source.

In Experiments 1, 3, and 5, observers were shown dynamic patterns of image shading. Three conditions were compared (see Figure 1). In the control condition, a simulated surface was rotated while the illumination source was kept stationary; in the in-phase condition, the surface was rotated in the same direction as the illumination source; in the counter-phase condition, the surface and the illumination source were rotated in opposite directions. Observers were asked to report the direction and the magnitude of the 3-D rotation of the simulated surface. In Experiments 2 and 4, observers were shown static patterns of image shading consisting of a subset of the images used to generate the apparent-motion sequences of the experiments mentioned before.

We found that the direction of surface rotation was recovered more accurately from static than from dynamic image shading. In the dynamic displays, performance was veridical in the control and the in-phase conditions, but it was systematically biased in the counter-phase condition. Conversely, no systematic biases were found when the im-

\section{Control Condition}
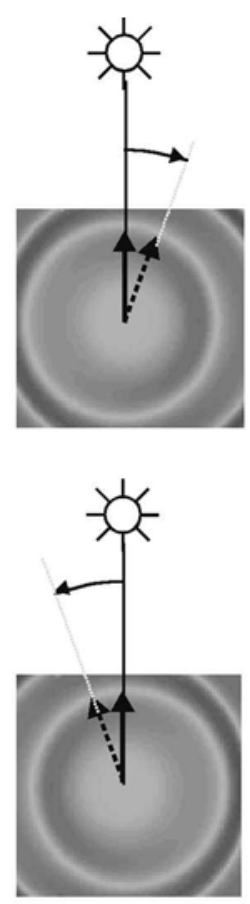
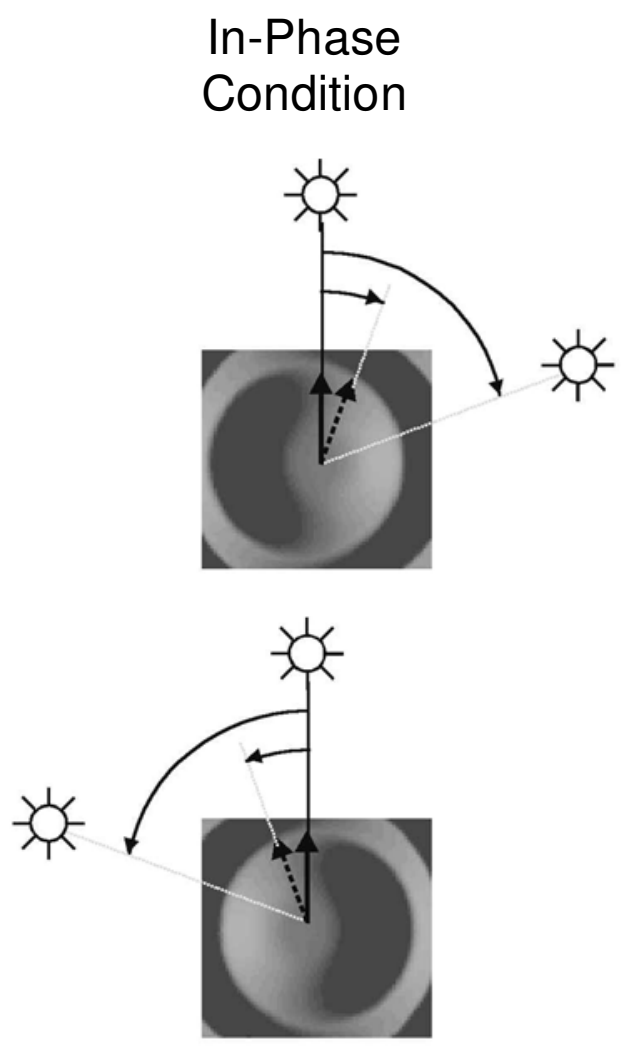

\section{Counter-Phase Condition}
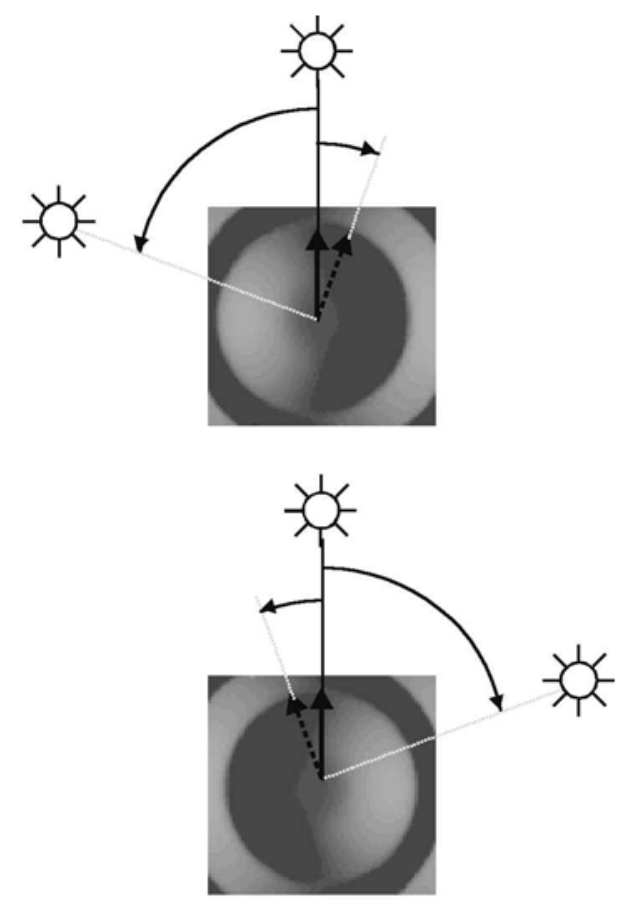

Figure 1. Each of the six images depicts (the central part of) the last frame of the stimulus sequences of the control (left panels), in-phase (central panels), and counter-phase (right panels) conditions of Experiment 1. The surface was simulated with positive $z$-depth values and was rotated about the vertical axis in the counterclockwise (top panels) and clockwise (bottom panels) directions. (See Experiment 1 for a full description of the stimulus parameters.) 
ages used for the dynamic displays were presented in isolation (as static images). In Experiment 6, finally, the biases found with the computer simulations of the previous experiments were replicated with solid objects.

\section{EXPERIMENT 1}

The transformations of the luminance gradients caused by a varying illuminant direction are known to induce local distortions in the 3-D shape perceived in static images (e.g., Christou \& Koenderink, 1997). The purpose of the present experiment was to establish whether, in dynamic images, the changing orientation of the illumination source can bias the perceived 3-D rotation of smoothly curved surfaces also.

\section{Method}

Participants. Nine University of Trieste undergraduates participated in this experiment. All of them were naive as to the purpose of the experiment. All the participants had normal or corrected-to-normal vision. They were not paid for their participation.

Apparatus. The displays were presented on a high-resolution color monitor $(1,280 \times 1,024$ addressable locations $)$ under the control of a Silicon Graphics INDIGO 2 Workstation. The screen had a refresh rate of $70 \mathrm{~Hz}$ and was approximately photometric ally linearized. The graphic buffer was 8 bit deep (256 gray levels). The participants viewed the displays through a reduction screen that reduced the field of view to a circular area with an approximate diameter of $5^{\circ}$ of visual angle. The eye-to-screen distance was $1.1 \mathrm{~m}$.

Design. Surface rotation and illuminant direction were manipulated so as to define three within-subjects conditions. In the control condition, the simulated surface was rotated while the illumination source was kept stationary; in the in-phase condition, the surface was rotated in the same direction as the illumination source; in the counter-phase condition, the surface and the illumination source were rotated in opposite directions.

Stimuli. The stimuli consisted of 13 images of smoothly curved surfaces (see Figure 1). The surfaces were simulated by using a procedure similar to that of Todd and Reichel (1989). The displays were generated within a Cartesian coordinate system $(x, y, z)$ where $x$ and $y$ were aligned with the horizontal and vertical axes of the display screen and $z$ was orthogonal to them. The depth $z$ at each point $i$ of the surface was computed as

$$
z_{i}=k \cos \left(x_{i}^{2}+y_{i}^{2}\right),
$$

where $k$ is a constant. The surface was then rotated from this canonical position by $10^{\circ}$ (either in the clockwise direction, $\theta_{-10}$, or in the counterclockwise direction, $\theta_{10}$ ) about a vertical axis throughout the origin and displayed under parallel projection.

The intensity $\left(I_{x}\right)$ of each image point was determined by assuming a Lambertian reflection (i.e., light intensity was assumed to be the same over all viewing directions). By simulating an infinitely distant point-light source, $I_{x}$ was calculated with the following equation:

$$
I_{x}=k_{a} I_{a}+k_{d} I_{l}\left(\mathbf{N}_{x} \cdot \mathbf{L}\right),
$$

where $k_{a}$ is the ambient-reflection coefficient for modifying the ambient-light intensity $I_{a}, k_{d}$ is the surface's albedo, $I_{l}$ is the intensity of the point-light source, $\mathbf{N}_{x}$ is a unit normal vector to the surface, and $\mathbf{L}$ is the unit direction vector to the point light source from a position on the surface (see Pentland, 1982).

The total angle of $10^{\circ}$ of rotation about the vertical axis (in the clockwise or counterclockwise direction) was divided by the number of frames minus one so as to determine the incremental rotation $\Delta \vartheta$. Each of the 13 images of the apparent motion sequences was generated by rotating the simulated surface away from the canonical position by an angle equal to the number of the frame within the sequence (starting from 0 ) times $\pm \Delta \vartheta$. The illumination source was simulated to be either stationary $\left(\mathbf{L}_{0}\right.$, the illuminant direction was horizontally centered) or itself undergoing a rotation. The illuminant direction in each frame was defined by an angle equal to the number of the frame within the sequence (starting from 0) times $\pm 6.667^{\circ}$.

Six apparent motion sequences were generated. In the control condition, the illumination source was stationary $\left(\mathbf{L}_{0}\right)$, and the surface was simulated to rotate between $0^{\circ}$ (the canonical position) and $10^{\circ}$ $\left(\theta_{10}\right)$ or between $0^{\circ}$ and $-10^{\circ}\left(\theta_{-10}\right)$. In the in-phase condition, the surface was simulated as rotating counterclockw ise from $0^{\circ}$ to $10^{\circ}\left(\theta_{10}\right)$ while the illumination source rotated counterclockwise from $0^{\circ}$ to $80^{\circ}\left(\mathbf{L}_{80}\right)$. Alternatively, the surface was simulated to rotate clockwise from $0^{\circ}$ to $-10^{\circ}\left(\theta_{-10}\right)$ while the illumination source rotated clockwise from $0^{\circ}$ to $-80^{\circ}\left(\mathbf{L}_{-80}\right)$. In the counter-phase condition, the surface was simulated to rotate clockwise from $0^{\circ}$ to $-10^{\circ}\left(\theta_{-10}\right)$ while the illumination source rotated counterclockwise from $0^{\circ}$ to $80^{\circ}$ $\left(\mathbf{L}_{80}\right)$. Alternatively, the surface was simulated to rotate counterclockwise from $0^{\circ}$ to $10^{\circ}\left(\theta_{10}\right)$ while the illumination source rotated clockwise from $0^{\circ}$ to $-80^{\circ}\left(\mathbf{L}_{-80}\right)$.

The apparent-motion sequences were generated by displaying each frame with a stimulus onset asynchrony of $160 \mathrm{msec}$ and an interstimulus interval of 0 . Each image was contained in an area subtending $6.2^{\circ}$ of visual angle and was surrounded by a checkerboard pattern with irregular contours to minimize the information provided by the foreshortening of the outer contours of the simulated surface. An icon representing two intersecting line segments was shown in the upper part of the terminal screen. Movement of a mouse connected to the workstation varied the represented orientation of the icon. The angular magnitude represented by the icon before the participants' adjustments was randomly selected on each trial.

Procedure. All the participants were run individually in one session. Each participant viewed six presentations in random order of the six different stimuli. Six additional trials were presented at the beginning of each experimental session in order to familiarize the participants with the stimulus displays. The participants were asked (1) to report whether they perceived the surface as being convex or concave, (2) to report whether the surface appeared as being rotated in the clockwise or the counterclockwise direction, and (3) to report the maximum angle by which the surface appeared to be rotated away from its canonical position (i.e., $\theta_{0}$ ). The third task was performed by manipulating an icon present in the upper part of the terminal screen. The participants were told that the vertical line segment of the icon represented the side view of the image plane and that the other line segment represented the global orientation of the simulated surface relative to the display screen. A cardboard model was used to illustrate the experimental task. Vision was monocular, head motion was not restricted, and eye movements were permitted. During the experiment, the experimental room was dark. No restriction was placed on viewing time. No feedback was provided until after the experiment was completed.

\section{Results and Discussion}

The depicted shading patterns were mathematically ambiguous and, thus, perceptually multistable. The central region of Figure 1, top left panel, for example, could be seen as a bump illuminated from the right or as a dimple illuminated from the left. In order to obtain reliable judgments relative to the magnitude and direction of the perceived surface rotation, the settings of the observers were coded as follows. Suppose that a display was compatible with the projection of a clockwise rotation of a convex surface (dimple) or as the counterclockwise rotation of a concave surface (bump). If one of these two interpretations was chosen, the response 
was coded correct and the reported magnitude of perceived rotation was assigned a positive value. Conversely, if the interpretation was counterclockwise rotation of a convex surface or clockwise rotation of a concave surface, the response was coded incorrect and the reported magnitude of perceived rotation was assigned a negative value.

Percentages of correct responses and magnitudes of perceived rotation in each experimental condition are shown in Figure 2. The proportion of correct responses for each subject in each experimental condition was transformed by an arcsine transformation (Winer, 1971). A within-subjects analysis of variance (ANOVA) on the transformed data showed a significant effect of the condition factor $[F(2,16)=$ $\left.58.926, p<.001, \eta^{2}=.880\right)$. The in-phase and the counterphase conditions differed significantly $[F(1,8)=178.05$, $\left.p<.001, \eta^{2}=.957\right]$. The counter-phase condition differed significantly from the control condition $[F(1,8)=48.451$, $\left.p<.001, \eta^{2}=.864\right]$, whereas the phase condition did not $[F(1,8)=2.361$, n.s.]. It was shown by $t$ tests that the control $[t(8)=3.500, p<.01]$ and in-phase $[t(8)=16.738$, $p<.001]$ conditions were significantly above chance, whereas the counter-phase condition $[t(8)=-9.079, p<$ $.001]$ was significantly below chance.

A second analysis was performed on the signed magnitudes of perceived angular rotation (where negative values indicate reported directions of rotation incompatible with a mathematically correct analysis of the stimulus information). A repeated measures ANOVA showed that the effect of the condition variable was significant $[F(2,16)=$ $\left.17.029, p<.01, \eta^{2}=.680\right]$. The in-phase and counter-phase conditions differed significantly $[F(1,8)=17.982, p<.01$, $\left.\eta^{2}=.692\right]$. The in-phase condition differed significantly from the control condition $\left[F(1,8)=16.106, p<.01, \eta^{2}=.668\right]$.
The counter-phase condition differed significantly from the control condition $\left[F(1,8)=15.184, p<.01, \eta^{2}=.655\right]$. A $t$ test against the simulated value of $10^{\circ}$ revealed that, in the in-phase condition, the amount of surface rotation was significantly overestimated $[t(8)=2.697, p<.05]$. In the counter-phase condition, the perceived magnitude of surface rotation differed significantly from the simulated value in the wrong direction $[t(8)=-5.345, p<.001]$. In the control condition, finally, the amount of surface rotation was significantly underestimated $[t(8)=-2.608, p<$ $.05]$.

A third analysis also revealed a significant effect of the condition variable on the absolute magnitudes of perceived angular rotation $\left[F(2,16)=7.329, p<.05, \eta^{2}=.478\right]$. The in-phase condition $\left(21.1667^{\circ}, S E=4.141^{\circ}\right)$ differed significantly from the control condition $\left[6.426^{\circ}, S E=1.331^{\circ}\right.$; $\left.F(1,8)=11.600, p<.01, \eta^{2}=.592\right]$, and the counter-phase condition $\left(20.361^{\circ}, S E=5.681^{\circ}\right)$ differed significantly from the control condition $\left[F(1,8)=5.161, p<.05, \eta^{2}=.412\right]$.

In conclusion, these results indicate that the dynamic change of the illuminant direction affected perceived surface rotation. For the present stimulus conditions, the effect of the variation of the illuminant direction was very dramatic. Whereas in the control and in-phase conditions the perception of the direction of surface rotation was veridical (percent correct was $82 \%$ and $98 \%$, respectively), in the counter-phase condition accuracy dropped to only $6 \%$, thus revealing a systematic bias in the perception of surface rotation. In the counter-phase condition, a simulated clockwise rotation tended to be perceived as a counterclockwise rotation, and a simulated counterclockwise rotation tended to be perceived as a clockwise rotation. Even though, in the counter-phase condition, the illumi-

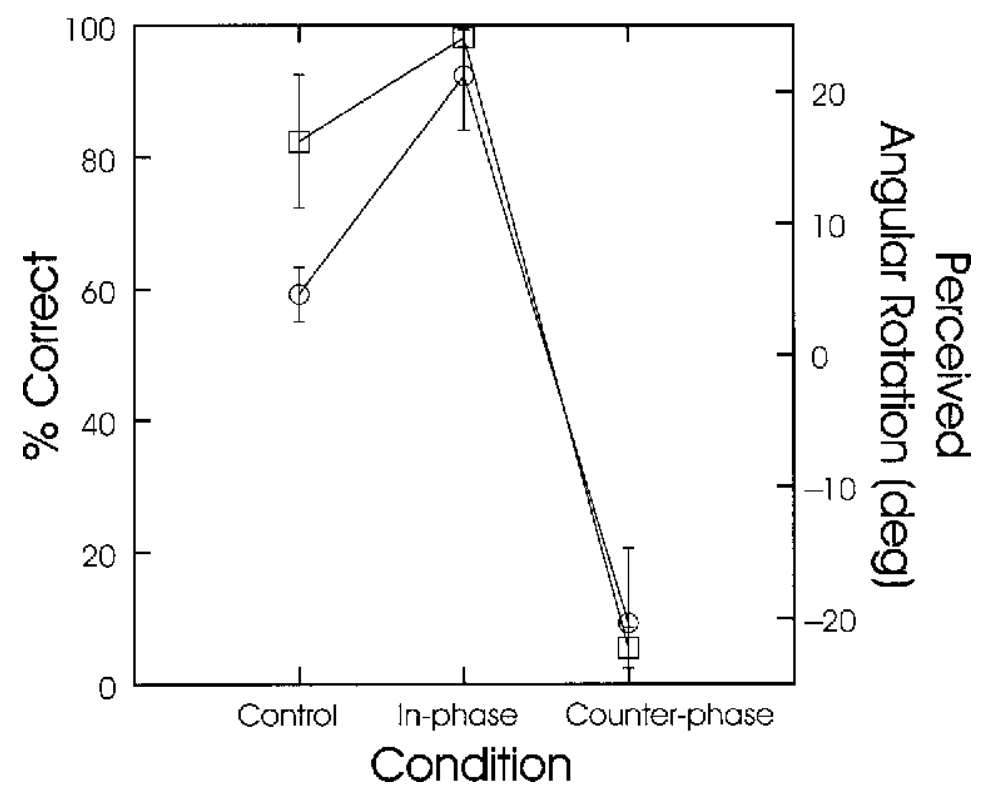

Figure 2. Mean judgments of surface rotation in Experiment 1. Squares represent percent correct, and circles represent the signed magnitude of perceived rotation. (Vertical bars represent $\pm 1 S E$.) 
nation source and the simulated surface were simulated as rotating in opposite directions, in the majority of the cases observers reported that the surface appeared to rotate in the same direction as the illumination source. Also affected by the dynamic variation of the illuminant direction was the magnitude of perceived surface rotation: Larger amounts of surface rotations were reported (in both the in-phase and the counter-phase conditions) when the illuminant was simulated as rotating, rather than being stationary.

\section{EXPERIMENT 2}

The previous results indicate that the perceived direction of surface rotation can be systematically biased by the counter-phase rotation of the illumination source. These results leave open the question of whether the reported misperceptions of surface rotation are due to the dynamic properties of the displays or can be found also when the luminance gradients used in the previous experiment are presented statically. To investigate this question, in each trial of Experiment 2, the observers were presented with a static image. These images were identical to the last frames of the stimulus sequences used in Experiment 1.

\section{Method}

Participants. Five University of Trieste undergraduates participated in this experiment. All of them were naive as to the purpose of the experiment. All the participants had normal or corrected-to-normal vision. They were not paid for their participation. None of them had participated in Experiment 1.

Apparatus. The apparatus was the same as that in Experiment 1.

Design. Surface orientation and illuminant direction were manipulated so as to define three within-subjects conditions. In the control condition the surface was rotated by $10^{\circ}$ or $-10^{\circ}$ away from its canonical position, and the illuminant direction was horizontally centered; in the in-phase condition, the surface orientation was $10^{\circ}$ and the illuminant direction was $80^{\circ}$, or the surface orientation was $-10^{\circ}$ and the illuminant direction was $-80^{\circ}$; in the counter-phase condition, the surface orientation was $10^{\circ}$ and the illuminant direction was $-80^{\circ}$, or the surface orientation was $-10^{\circ}$ and the illuminant direction was $80^{\circ}$.

Stimuli. The stimuli were six images depicting the smoothly curved surface defined in Experiment 1 under varying illumination conditions. There were three possible illuminant directions relative to the observers' line of sight: $\mathbf{L}_{0}$, where the illuminant direction was horizontally centered; $\mathbf{L}_{80}$, where the illuminant direction $\mathbf{L}_{0}$ was displaced by $80^{\circ}$ in the counterclockwise direction; and $\mathbf{L}_{-80}$, where the illuminant direction was displaced by $80^{\circ}$ in the clockwise direction. Three conditions were created. In the control condition, the illuminant direction was $\mathbf{L}_{0}$, and the simulated surface was rotated away from its canonical position by $10^{\circ}\left(\theta_{10}\right)$ or $-10^{\circ}\left(\theta_{-10}\right)$. In the inphase condition, the illuminant direction was $\mathbf{L}_{80}$ for $\theta_{10}$ or $\mathbf{L}_{-80}$ for $\theta_{-10}$; in the counter-phase condition, the illuminant direction was $\mathbf{L}_{80}$ for $\theta_{-10}$, or $\mathbf{L}_{-80}$ for $\theta_{10}$. In each trial, only one static image was shown. All the other stimulus parameters were identical to those used in Experiment 1.

Procedure. The procedure and instructions were the same as those in Experiment 1, except that the participants were asked to judge the global orientation of static 3-D surfaces.

\section{Results and Discussion}

The judgments of the participants were coded by using the same procedure as that in Experiment 1. Overall, per- formance was very accurate: Percent correct was $98 \%$ in the in-phase condition, $95 \%$ in the counter-phase condition, and $81 \%$ in the control condition (see Figure 3 ). The proportion of correct responses for each subject in each condition was transformed by an arcsine transformation. A repeated measures ANOVA performed on the transformed data revealed that the condition factor was not significant $\left[F(2,8)=8.429, p<.05, \eta^{2}=.678\right]$. It was shown by $t$ tests that the judgments of surface orientation were significantly above chance for the in-phase $[t(4)=11.205$, $p<.001]$, counter-phase $[t(4)=7.240, p<.01]$, and control $[t(4)=3.102, p<.05]$ conditions.

The signed magnitude of the perceived angular displacement was also analyzed. The observers reported a global orientation of $7.067^{\circ}$ in the in-phase condition, $7.433^{\circ}$ in the counter-phase condition, and $7.234^{\circ}$ in the control condition. A repeated measures ANOVA revealed that these differences were not statistically significant $[F(2,8)=0.02$, n.s.]. It was shown by $t$ tests performed against the simulated value of $10^{\circ}$ that surface orientation was significantly underestimated in the in-phase condition $[t(4)=-5.10$, $p<.01]$ but that the difference was not statistically significant in the counter-phase $[t(4)=-1.514$, n.s. $]$ and control $[t(4)=-1.336$, n.s. $]$ conditions .

The purpose of Experiment 2 was to determine whether, in static images, the perceived global orientation of the simulated surfaces can be affected by changing the illuminant direction. Even though the luminance gradients in the stimulus displays were dramatically altered by the variation of the illuminant direction, the present results indicate that perceived surface orientation was not significantly biased by changing the illuminant direction over a $160^{\circ}$ range. Even if the magnitude of angular displacement was slightly underestimated, the judgments of perceived surface orientation were compatible with a veridical analysis of the stimulus information, regardless of the relative orientation between the surface and the illuminant direction. The results of Experiment 2, therefore, are very different from those obtained in Experiment 1, even though the relative orientation between the illuminant direction and the surface in the last frame of the displays of Experiment 1 was identical to the relative orientation between the illuminant direction and the surface in the displays of Experiment 2 . Since the animated sequences of Experiment 1 simply added more views to the single images shown in each trial of Experiment 2 (and since, in those additional views, the illuminant direction was less extremely deviant from the normal to the $x-y$ plane than in Experiment 2), the present results indicate that the bias observed in the counter-phase condition of Experiment 1 cannot be attributed to the static properties of the luminance gradients. Instead, those biases must be attributed to the dynamic properties of the displays.

\section{EXPERIMENT 3}

The possibility of generalizing the results of the previous experiments beyond the specific stimulus parameters 


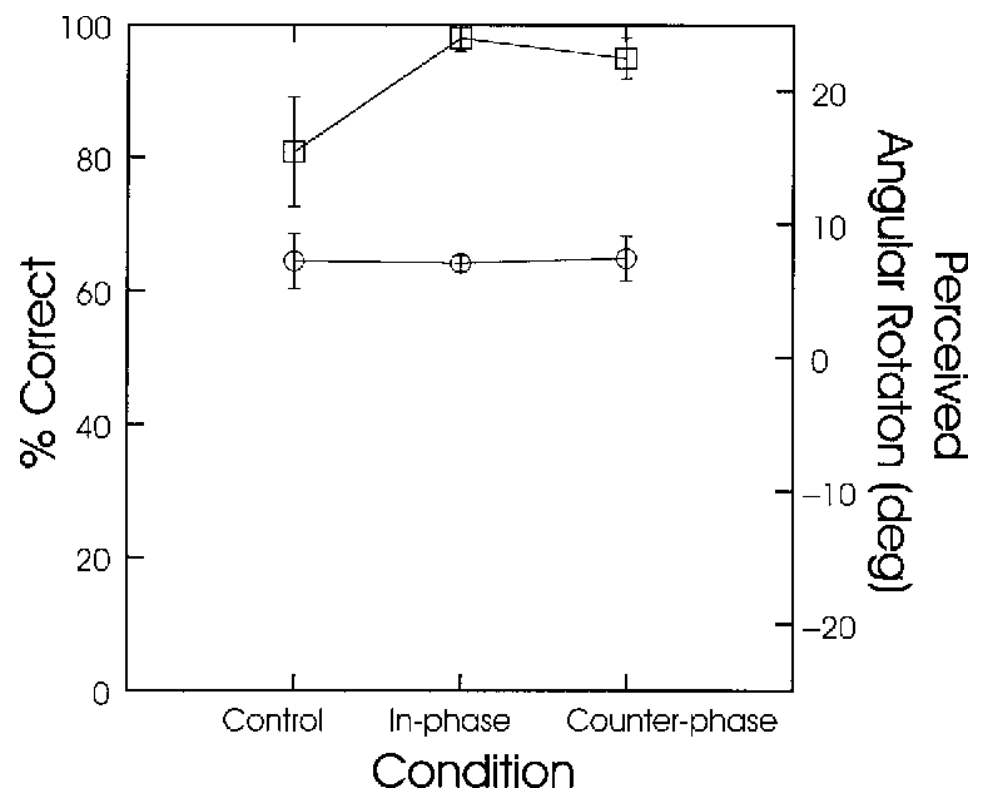

Figure 3. Mean judgments of global surface orientation in Experiment 2. Squares represent percent correct, and circles represent the signed magnitude of perceived rotation. (Vertical bars represent $\pm 1 S E$.)

that have been employed is limited by two factors. The first problem concerns the fact that, in Experiments 1 and 2 , the displays were generated by relying solely on surface normals and cast shadows were not computed. The resulting shading patterns of these experiments, therefore, were physically impossible when the illumination source was rotated by $80^{\circ}$. For such extreme rotations, in fact, the light would have to pass through solid matter for any of it to fall on the visible surface regions of the simulated stimuli. In the previous experiments, cast shadows were not computed so as to isolate shading information as the only cue to 3-D shape. However, it is reasonable to be suspicious about drawing any general conclusions from such ecologically unnatural displays, since several demonstrations have shown that human observers can successfully interpret shadow deformations produced both by the observer's motion and by the motion of the objects or the illumination source (Blake \& Bülthoff, 1990, 1991; Bülthoff \& Mallott, 1988; Norman \& Todd, 1994; Todd, 1985; see also Cavanagh \& Leclerc, 1989; Kersten, Mamassian, Knill, \& Bülthoff, 1996; Knill, Mamassian, \& Kersten, 1997). A second problem concerns the use of a Lambertian illumination model. One may ask, in fact, whether observers would experience the same biases as those previously reported if a more realistic illumination model had been simulated.

The intent of Experiment 3 was to address these two issues by simulating more realistic patterns of shading (e.g., Christou \& Koenderink, 1997; Johnston \& Curran, 1996; Koenderink \& van Doorn, 1983), in which (1) appropriate cast shadows were provided and (2) the Lambertian illu- mination model was complemented with mutual illumination (Forsyth \& Zisserman, 1990) and mirror components.

\section{Method}

Participants. Sixteen University of Trieste undergraduates participated in this experiment. All of them were naive as to the purpose of the experiment. All the participants had normal or corrected-tonormal vision. They were not paid for their participation. None of them had participated in the previous experiments.

Apparatus. The apparatus was the same as that in Experiment 1.

Design. Two independent variables were studied in this experiment: the within-subjects condition variable (control, in-phase, and counter-phase), defined as in the previous experiments, and the between-subjects illumination model variable (Lambertian, Lambertian plus mutual illumination component, Lambertian plus mutual illumination and mirror components).

Stimuli. Each stimulus display consisted of 11 images depicting a smoothly curved surface. The $z$-depth of the simulated surface was computed as follows:

$$
\left\{\begin{array}{l}
\left|x_{i}\right|<x_{m} ;\left|x_{i}\right|>3 x_{m} \rightarrow z_{i}=0 \\
x_{m} \leq\left|x_{i}\right| \leq 3 x_{m} \rightarrow z_{i}=\sin \left(90 \frac{\left|x_{i}\right|-x_{m}}{x_{m}}\right) z_{m}
\end{array}\right.
$$

where $2 x_{m}$ represents the amplitude of the two "bumps" on the $x$ axis and $z_{m}$ represents the maximum $z$-depth of the surface. Five vertical stripes were defined, the odd ones being flat and the even ones being modulated as a convex sinusoidal surface (on the $x-z$ plane). The surface was then rotated from this canonical position by $15^{\circ}$ (in the clockwise or counterclockwise direction) about the vertical axis and displayed under parallel projection. The total angle of rotation $\left(60^{\circ}\right)$ was divided by the number of frames minus one so as to determine the incremental rotation $\Delta \vartheta$. Each of the 11 images of the apparent motion sequences of Experiment 2, therefore, was generated by rotating the simulated surface away from its canonical posi- 
tion by an angle equal to the number of the frame within the sequence (starting from 0 ) times $\pm \Delta \vartheta$. The illumination source was simulated to be either stationary $\left(\mathbf{L}_{0}\right.$, where the illuminant direction was horizontally centered) or itself undergoing a rotation. The illuminant direction in each frame was defined by an angle equal to the number of the frame within the sequence (starting from 0 ) times $\pm 6^{\circ}$.

As in the previous experiment, six apparent-motion sequences were generated. The control, in-phase, and counter-phase conditions were defined as previously, the only difference being that the illumination source was rotated by $60^{\circ}$ from the straight-on position, rather than by $80^{\circ}$, and the surface was rotated by $15^{\circ}$ from the canonical position, rather than by $10^{\circ}$.

The intensity of each image point was determined by using three different illumination models. The Lambertian model was defined as in Experiment 1, apart from the geometrical factor Shadow:

$$
I_{x}=k_{a} I_{a}+\operatorname{Shadow}(x) k_{d} I_{l}\left(\mathbf{N}_{x} \cdot \mathbf{L}\right),
$$

where the function Shadow takes on the value of 1 or 0 , depending on whether the patch received direct light or not. The Lambertian model with a mutual illumination component (Christou \& Koenderink, 1997) was defined as

$$
\begin{aligned}
I_{x}= & k_{a} I_{a}+\operatorname{Shadow}(x) k_{d} I_{l}\left(\mathbf{N}_{x} \cdot \mathbf{L}\right) \\
& +k_{d} \sum l_{\left(x^{\prime}\right)} k\left(x, x^{\prime}\right),
\end{aligned}
$$

where

$$
k\left(x, x^{\prime}\right)=\frac{\left[\mathbf{N}_{x} \cdot(-\mathbf{r})\right]\left[\mathbf{N}_{x^{\prime}} \cdot \mathbf{r}\right] \operatorname{View}\left(x, x^{\prime}\right)}{(\mathbf{r} \cdot \mathbf{r})^{2}}
$$

is the geometric form-factor (Hottel \& Sarofim, 1967) and represents the amount of light from the patch $x$ to the patch $x^{\prime}$.The function View takes on the value of 0 or 1, depending on whether $x$ and $x^{\prime}$ "see each other" or not (Christou \& Koenderink, 1997). Finally, the Lambertian model with mutual illumination and mirror components (Todd \& Mingolla, 1983) was defined as

$$
\begin{aligned}
I_{x}= & k_{a} I_{a}+\operatorname{Shadow}(x) k_{d} I_{l}\left(\mathbf{N}_{x} \cdot \mathbf{L}\right) \\
& +k_{d} \sum l_{\left(x^{\prime}\right)} k\left(x, x^{\prime}\right)+k_{g} I_{l}\left(\mathbf{N}_{x} \cdot \mathbf{H}\right)^{n},
\end{aligned}
$$

where $k_{g}$ represents the proportion of reflected light in the direction of the maximum highlight (the value of 1 represents a perfect mirror), $n$ represents the sharpness factor (Todd \& Mingolla, 1983), and $\mathbf{H}$ is a unit vector that bisects the angle between $\mathbf{V}$ and $\mathbf{L}$. $\mathbf{V}$ is a unit vector in the viewing direction, and $\mathbf{L}$ is a unit vector in the direction of the light source. To simplify the illumination model, the computation of the mutual illumination did not take into account the mirror component (see also Christou \& Koenderink, 1997).

Procedure. All the participants were run individually in one session. Each participant viewed four presentations in random order of the six different stimuli. In a training block at the beginning of each experimental session, the participants viewed $\mathrm{a} \pm 5^{\circ}, \pm 15^{\circ}, \pm 20^{\circ}$, and $\pm 25^{\circ}$ rotation of the simulated surface with a stationary illumination source (in random order). Feedback was given at the end of each trial of the training session (the participants were told the simulated direction and amount of surface rotation). In each trial, the frame sequence defining a stimulus display was shown three times, with an interval of $3 \mathrm{sec}$. The observers viewed the displays monocularly through a reduction screen with a window $6 \mathrm{~cm}$ high and $8 \mathrm{~cm}$ wide at a distance of $1 \mathrm{~m}$. The judgments were provided by adjusting the orientation of a cardboard model of the simulated surface. Otherwise, instructions and procedure were the same as those in the previous experiments.

\section{Results and Discussion}

The responses of the observers were coded as in the previous experiments. Percentages of correct responses in each experimental condition are shown in Figure 4.

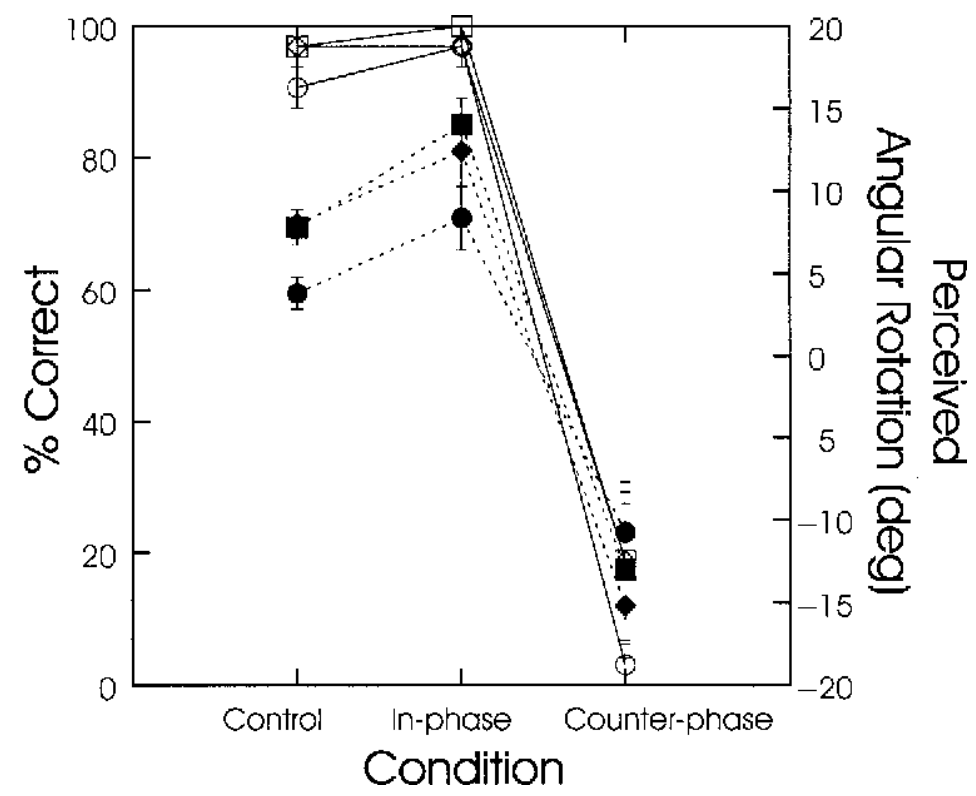

Figure 4. Mean judgments of surface rotation in Experiment 3. Open symbols represent percent correct; full symbols represent the signed magnitude of perceived rotation. Circles, Lambertian model; squares, Lambertian model with a mutual illumination component; diamonds, Lambertian model with mutual illumination and mirror components. (Vertical bars represent $\pm 1 S E$.) 
A 3 (condition: control, in-phase, and counter-phase) $\times 3$ (illumination model: Lambertian, Lambertian plus mutual illumination component, and Lambertian plus mutual illumination and mirror components) ANOVA on the transformed arcsine proportions of correct responses showed a significanteffect of the conditionfactor $[F(2,18)=162.912$, $\left.p<.001, \eta^{2}=.948\right]$. Percent correct was $95 \%$ for the control condition, $98 \%$ for the in-phase condition, and only $14 \%$ for the counter-phase condition. A comparison with the control condition revealed a significant difference for the counter-phase condition $\left[F(1,9)=187.585, p<.001, \eta^{2}=\right.$ $.954]$, but not for the in-phase condition $[F(1,9)=1.800$, n.s.]. The in-phase and the counter-phase conditions differed significantly $\left[F(1,9)=254.525, p<.001, \eta^{2}=.966\right]$. The illumination model variable was not significant $[F(2,9)=1.479$, n.s. $]$, and neither was the interaction between illumination model and condition $[F(4,18)=0.628$, n.s.]. It was indicated by $t$ tests that the control $[t(11)=$ $11.818, p<.001]$ and the in-phase $[t(11)=17.859, p<.001]$ conditions were significantly above chance, whereas the counter-phase condition was significantly below the value of chance $[t(11)=-5.947, p<.001]$.

The signed magnitude of perceived rotation was also analyzed. A repeated measures ANOVA, with condition as the within-subjects variable and illumination model as the between-subjects variable, showed a significant effect of the condition variable $\left[F(2,18)=62.174, p<.001, \eta^{2}=.874\right]$. Neither the illumination model variable $[F(2,9)=0.435$, n.s. $]$ nor the interaction between condition and illumination model $[F(4,18)=0.942$, n.s. $]$ was significant. The in-phase condition differed significantly from the control condition $\left[F(1,9)=32.171, p<.001, \eta^{2}=.781\right]$. The counter-phase condition differed significantly from the control condition $\left[F(1,9)=55.903, p<.001, \eta^{2}=.861\right]$. The in-phase and counter-phase conditions differed significantly $[F(1,9)=$ $\left.32.171, p<.001, \eta^{2}=.781\right]$. It was shown by $t$ tests against the simulated value of $15^{\circ}$ that perceived surface rotation was significantly underestimated in the control $[t(11)=$ $-11.067, p<.001]$ and in-phase $[t(11)=-2.774, p<.05]$ conditions. Average reported rotation was, respectively, $6.510^{\circ}\left(S E=0.767^{\circ}\right)$ and $11.562^{\circ}\left(S E=1.239^{\circ}\right)$. With an average reported rotation of $-13.312^{\circ}\left(S E=2.400^{\circ}\right)$, the counter-phase condition also differed significantly from the simulated value of $15^{\circ}[t(11)=-11.797, p<.001]$.

An analysis on the absolute values of the reported magnitudes of angular rotation revealed that the condition factor was significant $\left[F(2,18)=5.111, p<.05, \eta^{2}=.362\right]$ and that both the in-phase $\left[F(1,9)=32.171, p<.001, \eta^{2}=.781\right]$ and the counter-phase $\left[F(1,9)=6.713, p<.05, \eta^{2}=.427\right]$ conditions differed significantly from the control condition.

In conclusion, the results of Experiment 3 replicated those of Experiment 1. In both the control and the in-phase conditions, the perception of the direction of surface rotation was veridical; in the counter-phase condition, on the other hand, in the majority of the cases the observers reported that the surface appeared to rotate in the same direction as the illumination source, even though the illumination source and the surface were simulated as rotating in opposite directions. The findings of Experiment 3, therefore, indicate that the same misperceptions as those reported in Experi-

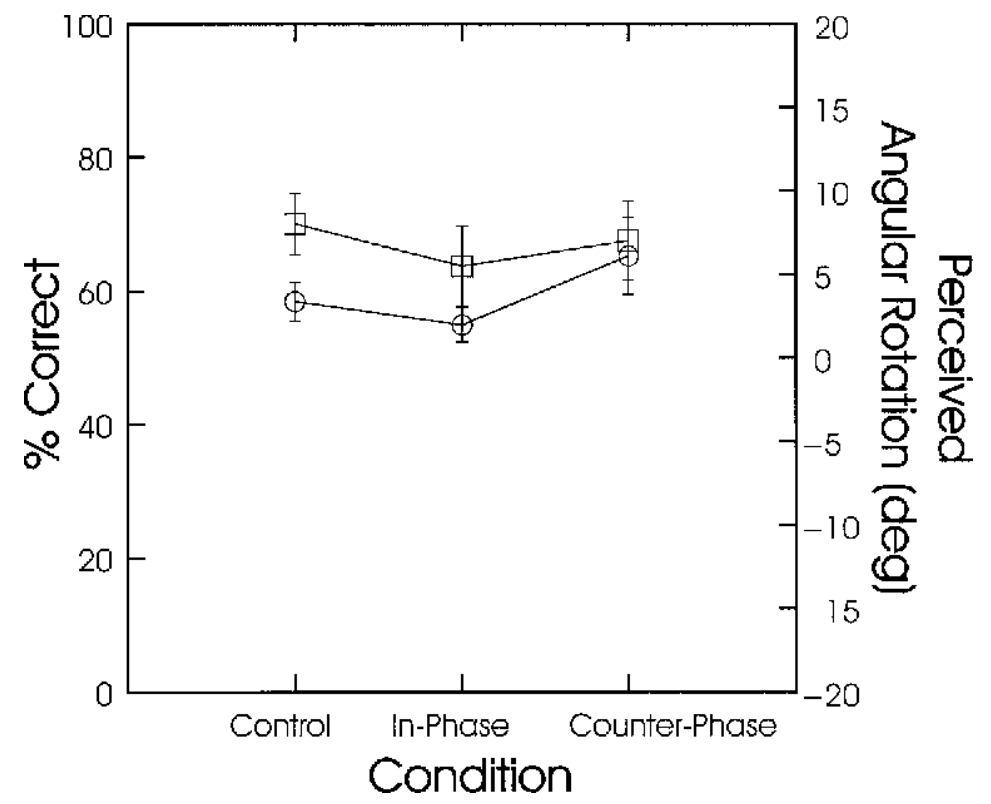

Figure 5. Mean judgments of global surface orientation in Experiment 4. Squares represent percent correct, and circles represent the signed magnitude of perceived rotation. (Vertical bars represent $\pm 1 S E$.) 
ment 1 can be evoked also by stimulus displays generated with a more realistic illumination model comprising cast shadows, mutual illumination, and mirror components.

\section{EXPERIMENT 4}

The purpose of Experiment 4 follows the same rationale as that for Experiment 2. Our hypothesis was that the last frames of the stimulus sequences used in Experiment 3, when presented in isolation, allow a veridical interpretation of the global orientation of the simulated surfaces, contrary to what happens when the same images are presented within an animated sequence.

\section{Method}

Participants. Ten University of Trieste undergraduates participated in this experiment. All of them were naive as to the purpose of the experiment. All the participants had normal or corrected-to-normal vision. They were not paid for their participation. None of them had participated in the previous experiments.

Apparatus. The apparatus was the same as that in Experiment 1.

Design. The design was the same as that in Experiment 2.

Stimuli. The stimuli consisted of six images, each corresponding to the last frames of the stimulus sequences used in Experiment 3. Only the displays generated with the more realistic illumination model (Lambertian model plus mutual illumination and mirror components) were used.

Procedure. The procedure and instructions were the same as those in Experiment 2.

\section{Results and Discussion}

Percent correct and mean perceived angular rotation in each experimental condition are shown in Figure 5. A repeated measures ANOVA performed on the arcsine transformed proportions of correct responses did not show a significant effect of the condition variable $[F(2,18)=0.155$, n.s.]. In all three experimental conditions, the judgments of global surface orientation were in the veridical direction. Percent correct was $70 \%$ in the control condition, $63.7 \%$ in the in-phase condition, and $67.5 \%$ in the counterphase condition. A $t$ test showed that all three conditions were significantly above chance [control, $t(9)=4.311, p<$ .01 ; in-phase, $t(9)=2.283, p<.05$; counter-phase, $t(9)=$ $2.941, p<.05]$.

A repeated measures ANOVA on the signed magnitudes of surface orientation did not reveal a significant effect for the condition variable $[F(2,18)=1.653$, n.s.]. In all three experimental conditions, mean judgments of surface orientation were in the veridical direction: $3.362^{\circ}$ (control condition), $1.975^{\circ}$ (in-phase condition), and $6.100^{\circ}$ (counterphase condition). Surface orientation, however, was clearly underestimated. A $t$ test showed that all three conditions were significantly below the simulated value of $15^{\circ}$ [control, $t(9)=-10.098, p<.001$; in-phase, $t(9)=-12.709$, $p<.001$; counter-phase, $t(9)=-3.862, p<.01]$.

In conclusion, the results of Experiment 4 replicated those of Experiment 2 with a more realistic illumination model. Even if the magnitude of surface rotation was underestimated, the judgments of global surface orientation were compatible with a veridical analysis of the stimulus information, regardless of the relative orientation between surface orientation and illuminant direction.

\section{EXPERIMENT 5}

The illuminant direction in the previous experiments was largely deviant from the normal to the image plane. In Experiments 1 and 2 , the angle defining the illuminant direction was $80^{\circ}$, and in Experiments 3 and 4 this angle was $60^{\circ}$. It is therefore of interest to establish whether the direction of surface rotation would be misperceived also for smaller angles of illuminant rotation. In Experiment 5, this problem was addressed by manipulating the amount of rotation of the illumination source.

\section{Method}

Participants. Twenty University of Trieste undergraduates participated in this experiment. All of them were naive as to the purpose of the experiment. All the participants had normal or corrected-tonormal vision. They were not paid for their participation. None of them had participated in the previous experiments.

Apparatus. The apparatus was the same as that in Experiment 1.

Design. Two independent variables were studied in this experiment. The amount of illuminant rotation was manipulated within subjects, and the absolute $z$-depth of the simulated surface was manipulated between subjects.

Stimuli. A stimulus trial consisted of an animated sequence representing the rotation of a surface while the illuminant source was itself rotating. The simulated surface shape was the same as that in Experiments 3 and 4, but two different maximum $z$-depth magnitudes were used. One surface had the same depth as in Experiments 3 and 4; the second surface was 50\% shallower. Each surface was rotated by $15^{\circ}$ or $-15^{\circ}$. When the surface was simulated as undergoing a clockwise rotation $\left(-15^{\circ}\right)$, the illumination source was simulated as undergoing a rotation of $-10^{\circ}, 0^{\circ}, 10^{\circ}, 20^{\circ}, 30^{\circ}, 40^{\circ}, 50^{\circ}$, $60^{\circ}, 70^{\circ}$, or $80^{\circ}$; when the surface was simulated as undergoing a counterclock wise rotation $\left(15^{\circ}\right)$, the illumination source was simulated as undergoing a rotation of $10^{\circ}, 0^{\circ},-10^{\circ},-20^{\circ},-30^{\circ},-40^{\circ},-50^{\circ},-60^{\circ}$, $-70^{\circ}$, or $-80^{\circ}$. The 20 conditions so defined were shown eight times within a random sequence to each observer. The displays were generated by using a Lambertian illumination model with mutual illumination and mirror components. Otherwise, the stimulus parameters were the same as those in Experiment 3.

Procedure. The procedure and instructions were the same as those in Experiment 3.

\section{Results and Discussion}

The average proportions of correct judgments for each experimental condition are reported in Figure 6. A probit analysis was used to calculate the point of subjective equality (PSE) - that is, the magnitude of illuminant rotation corresponding to a .5 probability that the direction of surface rotation would be reported correctly. Two observers out of 20 ( 1 in each group) were correct in more than $50 \%$ of the trials in each experimental condition (in other words, they never misperceived the direction of surface rotation). The magnitude of their PSEs was outside the permissible range of values that the independent variable could assume, so these data were excluded from the following analysis. A $t$ test comparing the remaining PSEs revealed a sig- 


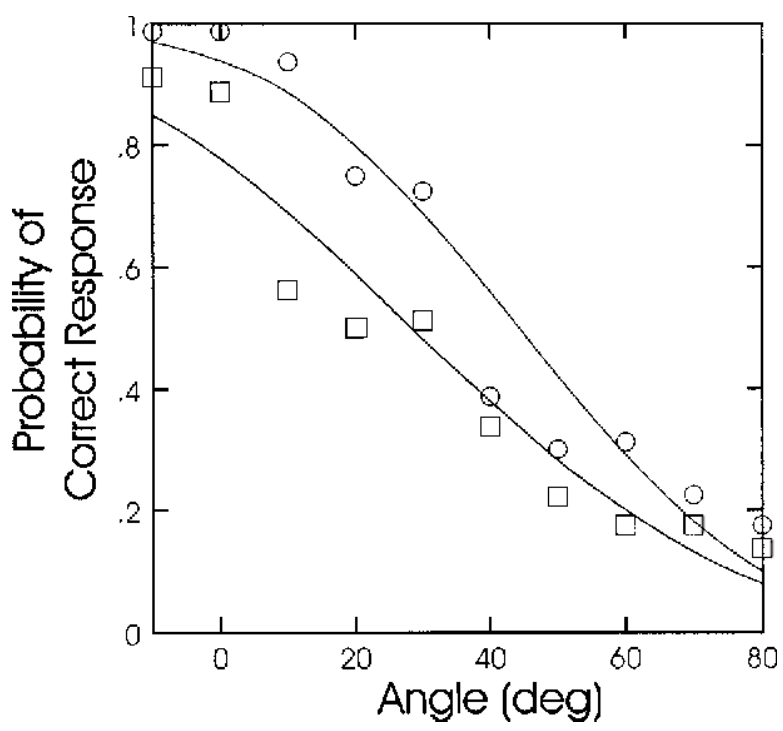

Figure 6. Mean proportions of correct responses in each condition of Experiment 5. Larger and smaller simulated $z$-depth magnitudes are represented by circles and squares, respectively. The lines represent a global (probit) fit to the data.

nificant difference between the two groups identified by the between-subjects independent variable $[t(16)=1.889$, $p<.05]$. The average PSEs for the deeper and the shallower surfaces were, respectively, $40.525^{\circ}\left(S E=5.810^{\circ}\right)$ and $23.061^{\circ}\left(S E=7.189^{\circ}\right)$.

The present results indicate, therefore, that the PSE depends on the $z$-depth magnitudes of the simulated surface: the shallower the surface, the smaller the PSE. Within the present stimulus parameters, for those observers that experienced this phenomenon, we found that a systematic bias in the perceived direction of surface rotation was evoked by a (counter-phase) rotation of the illuminant as small as $23^{\circ}$.

\section{EXPERIMENT 6}

In Experiment 6, solid objects were used, and judgments about the perceived direction of their 3-D rotations were collected under a stationary or a moving source of illumination. The purpose of the experiment was to determine whether the same biases found in the computer simulations of the previous experiments can also be found within a natural setting.

\section{Method}

Participants. Twelve University of Trieste undergraduates participated in this experiment. All of them were naive as to the purpose of the experiment. All the participants had normal or corrected-tonormal vision. They were not paid for their participation. None of them had participated in the previous experiments.

Apparatus. A viewing box was assembled, so that a target object could be seen through a pinhole. The dimensions of the viewing box are indicated in Figure 7. A dim light source $(10 \mathrm{w})$ was positioned on a rail and could be rotated within the box by about $24^{\circ}$, maintaining a fixed distance of $1 \mathrm{~m}$ relative to the target object. The inner walls of the box were painted black.

Design. Only two within-subjects conditions were defined: a moving illumination source (MIS) condition and a stationary illumination source (SIS) condition.

Stimuli. The test object was made up of a sheet of glossy white paper (16 cm wide and $7 \mathrm{~cm}$ high) that was bent in such a manner as to create a convex bump with an approximately sinusoidal profile (see Figure 7). The stimulus was always stationary. In the MIS condition, the illumination source was rotated from one extreme of the rail to the other, back and forth three times. In the SIS condition, the illumination source was kept stationary at one or the other extreme end of the rail.

Procedure. The experimental task was to report (1) whether the test surface was perceived to be convex or concave and (2) whether the test surface was perceived to rotate in the clockwise or the counterclockwise direction. The observers were told to provide their judgments about the direction of rotation in the first half-cycle of the displacement of the light source. For each observer, eight judgments were collected in the SIS condition, and eight judgments were collected in the MIS condition. The SIS condition always preceded the MIS condition. At the beginning of each trial, the light source was positioned either at the extreme right or at the extreme left of the rail. These two end positions were equally represented in each session, with the order of presentation randomized for each participant. A cardboard model was used to illustrate the experimental task. Vision was monocular, head motion was restricted with a chinrest, and eye movements were permitted. During the experiment, the experimental room was dark. The participants provided their judgments after the stimulus had been shown for approximately $6 \mathrm{sec}$. No feedback was provided until after the experiment was completed.

\section{Results and Discussion}

The purpose of the experiment was to determine whether the motion of the illumination source would induce a bias in the responses of the observers. Since the test object was

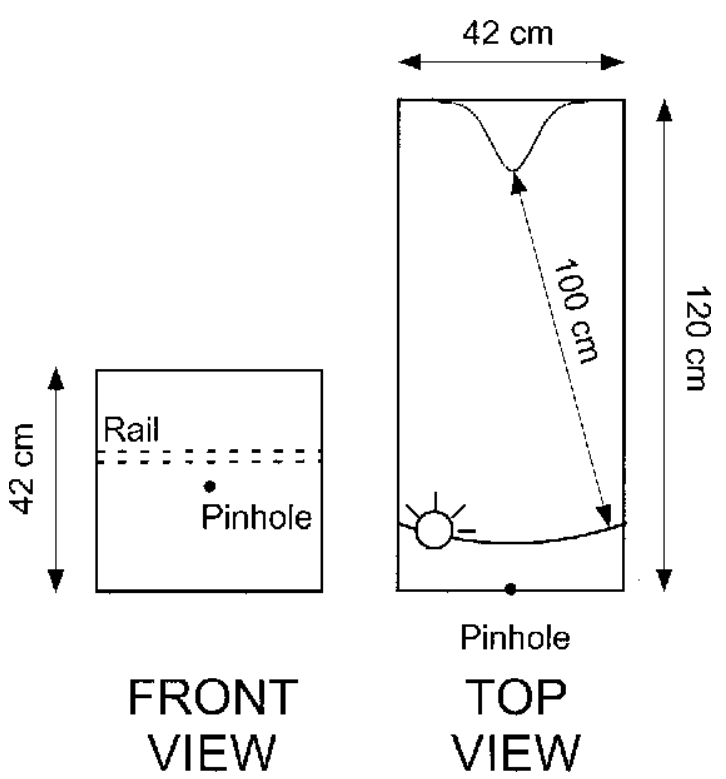

Figure 7. Schematic representation of the apparatus used in Experiment 6. 


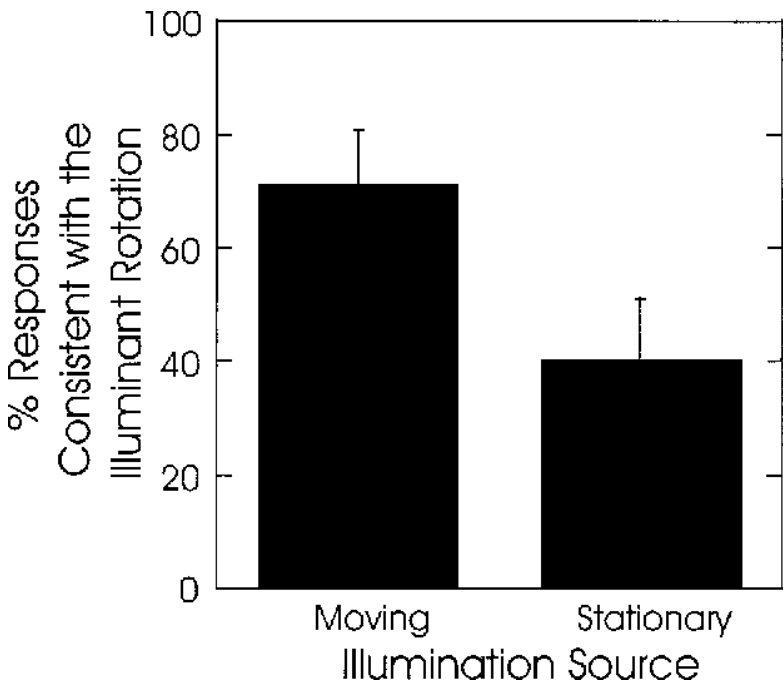

Figure 8. Mean proportions of responses consistent with the illuminant rotation. (Vertical bars represent $\pm 1 S E$.)

always stationary, the responses of the participants could not be coded as correct or incorrect. If the test object was reported to rotate in the same direction as the illumination source, the responses were coded as consistent with the illuminant rotation; otherwise, the responses were coded as inconsistent with the illuminant rotation. The proportions of responses consistent with the illuminant rotation in the two experimental conditions are shown in Figure 8.

In the SIS condition, the proportion of responses consistent with the illuminant rotation did not differ significantly from chance $[t(11)=-.987$, n.s. $]$. This indicates that no bias is shown when the illumination source is stationary. On the other hand, a $t$ test for paired observations showed a significant difference between the two conditions $[t(11)=3.252, p<.01]$. Consistent with the hypothesis that motivated the present experiment, in most of the cases, the observers in the MIS condition reported that the test object appeared to rotate in the same direction as the illumination source. This result indicates, therefore, that the biases found in the computer-generated displays of the previous experiments can occur also for solid objects under real illuminants (see also Johnston \& Curran, 1996).

\section{GENERAL DISCUSSION}

The perception of a smoothly curved surface defined by a pattern of image shading was investigated in both dynamic (Experiments 1, 3, and 5) and static (Experiments 2 and 4) displays. Observers were asked to judge the magnitude and the direction of rotation of rotating surfaces (Experiments 1,3, and 5) or the global orientation (Experiments 2 and 4) of static surfaces. Under varying illuminant conditions, the 3-D orientation of the simulated surfaces and the illuminant direction were manipulated. We found that perceptual performance was poorer with dynamic than with static displays.
For static displays, the illuminant direction had a small influence on perceived global surface orientation, even if the illuminant direction varied over a large range $\left( \pm 80^{\circ}\right.$ in Experiment 2, and $\pm 60^{\circ}$ in Experiment 4). Regardless of the illuminant direction, global surface orientation was perceived veridically. For dynamic displays, on the other hand, the same variations of illuminant direction induced systematic biases in the perceived direction of surface rotation.

The dynamic displays were generated by simulating the continuous rotation about a vertical axis of the same surfaces as those used in the static displays. Three conditions were defined. In the control condition, the source of illumination was stationary, whereas in the other two conditions, it underwent a continuous change of location. In the inphase condition, both surface and illumination source rotated in the same direction. In the counter-phase condition, conversely, they rotated in opposite directions.

For dynamic displays, the direction of illuminant rotation strongly influenced the perceived direction of surface rotation. The direction of surface rotation was reported veridically in the control and in-phase conditions, but not in the counter-phase condition. In the counter-phase condition, the surface appeared to rotate in the same direction as the illumination source, even though the displays simulated a surface and an illumination source rotating in opposite directions.

The simplest hypothesis that may be proposed to explain the present misperceptions of dynamic image shading is the prior assumption of an invariant illuminant direction (e.g., Mamassian, Knill, \& Kersten, 1998). Let us suppose, for example, that the illumination source rotates in the clockwise direction while the surface remains stationary. According to the hypothesis of an invariant illuminant direction, in these circumstances the surface would be perceived as rotating in the counterclockwise direction. This prediction, however, does not account for the present data. In the counter-phase condition of the previous experiments, in fact, the observers reported that the surface appeared to rotate in the same direction as the illumination source, even though the surface and the illumination source were simulated as rotating in opposite directions.

The misperceptions found in Experiments 1, 3, and 5 cannot be directly compared with the distortions of perceived shape from shading discussed in the introduction. Whereas in those studies the manipulation of the illuminant direction induced local distortions in the 3-D shape perceived in static patterns of image shading, in our static images the manipulation of the illuminant direction did not significantly influence perceived global surface orientation. The new contribution of the present experiments is to show that dynamic image shading may evoke misperceptions of surface orientation even when a corresponding pattern of static image shading does not.

It is also interesting to compare the results of the present investigation with those obtained by Norman, Todd, and Phillips (1995). Regardless of the differences in the methodology that had been employed, in both investigations sta- 
tic and dynamic patterns of image shading were used. Whereas the focus of the present investigation was on the perception of dynamic shading generated by a moving illumination source, they studied the perception of dynamic shading generated by 3-D shapes rotating under a stationary illumination source. In their investigation, observers were asked to perform an orientation matching task on a randomly shaped 3-D object defined by Lambertian shading and specular highlights. Norman et al. found an improvement of local orientation judgments when the simulated objects were rotated in depth about a vertical axis by $\pm 12^{\circ}$, as opposed to when they were stationary. With a stationary illumination source and moving shaded objects, the overall level of performance was found to be only slightly worse than the performance obtained with textured objects. What should be noticed is that the stimuli used by Norman et al. are comparable to those used in our control condition (where we simulated a stationary or moving object under a stationary illumination source). Even if our experimental task was different from that of Norman et al., performance with static and dynamic displays can be compared also in the control conditions of our experiments. Where the illumination model was richer (in Experiments 3 and 4), we found that, in the dynamic displays, the errors (i.e., the absolute difference between the simulated and the reported magnitude of angular rotation) were smaller than in the static displays $\left(3.490^{\circ}\right.$ vs. $6.638^{\circ}$, respectively). This difference turned out to be statistically significant $[t(20)=2.341, p<.05]$, thus replicating the results of Norman et al. (a similar analysis performed on the data of Experiments 1 and 2 did not produce a significant result).

\section{CONCLUSION}

In the present paper, we investigated the perception of shape from shading for stimuli depicting smoothly curved surfaces with no visible occlusion contours. Perceptual performance with static shading displays was compared with performance with dynamic displays representing the rotation of the simulated surfaces under a stationary or a moving illumination source. For the static displays, the global orientation of the simulated surfaces was always correctly perceived. For the dynamic displays, performance was veridical when the illumination source was stationary, but not when it was moving. In particular, systematic biases were found in the dynamic displays when the surfaces were simulated to rotate in one direction and the illumination source was simulated to rotate in the opposite direction, even if in the corresponding static displays performance was veridical. The present results suggest that perceptual processing of dynamic shading may be mediated by a different mechanism than is perceptual processing of static shading.

\section{REFERENCES}

Blake, A., \& Bülthoff, H. (1990). Does the brain know the physics of specular reflection? Nature, 343, 165-168.

Blake, A., \& BÜlthoff, H. (1991). Shape from specularities: Compu- tation and psychophysics. PhilosophicalTransactions of the Royal Society of London: Series B, 331, 237-252.

Bülthoff, H., \& Mallott, H. A. (1988). Integration of depth modules: Stereo and shading. Journal of the Optical Society of America A, 5, 1749-1758.

Cavanagh, P., \& Leclerc, Y. G. (1989). Shape from shadows. Journal of Experimental Psychology: Human Perception \& Performance, 15, 3-27.

Christou, C. G., \& Koenderink, J. J. (1997). Light source dependence in shape from shading. Vision Research, 37, 1441-1449.

D'ZMurA, M. (1991). Shading ambiguity: Reflectance and illumination. In M. S. Landy \& J. A. Movshon (Eds.), Computationalmodels of visual processing (pp. 187-207). Cambridge, MA: MIT Press.

Erens, R. G. F., Kappers, A. M. L., \& Koenderink, J. J. (1993a). Estimating local shape from shading in the presence of global shading. Perception \& Psychophysics, 54, 334-342.

Erens, R. G. F., Kappers, A. M. L., \& Koenderink, J. J. (1993b). Perception of local shape from shading. Perception \& Psychophysics, 54, 145-156.

Forsyth, D. A., \& Zisserman, A. (1990). Shape from shading in the light of mutual illumination. Image \& Vision Computing, 8, 29-42.

HorN, B. K. P. (1975). Obtaining shape from shading information. In P. H. Wistron (Ed.), The psychology of computer vision (pp. 115-155). New York: McGraw-Hill.

Horn, B. K. P. (1977). Understanding image intensities. Artificial Intelligence, 8, 201-231.

Horn, B. K. P., \& Brooks, M. J. (1989). Shape from shading. Cambridge, MA: MIT Press.

Hottel, H. C., \& SARofim, A. D. (1967). Radiative transfer. New York: McGraw-Hill.

Johnston, A., \& Curran, W. (1996). Investigating shape from shading illusions using solid objects. Vision Research, 36, 2827-2835.

Johnston, A., \& Passmore, P. J. (1994a). Shape from shading: I. Surface curvature and orientation. Perception, 23, 169-190.

Johnston, A., \& Passmore, P. J. (1994b). Shape from shading: II. Geodesic bisection and alignment. Perception, 23, 191-200.

Kersten, D., Mamassian, P., Knill, D. C., \& Bülthoff, I. (1996). Illusory motion from shadows. Nature, 379, 31 .

Knill, D. C., Mamassian, P., \& Kersten, D. (1997). Geometry of shadows. Journal of the Optical Society of America A, 14, 3216-3232.

Koenderink, J. J., Kappers, A. M. L., Todd, J. T., Norman, J. F., \& Phillips, F. (1996). Surface range and attitude probing in stereoscopically presented dynamic scenes. Journal of Experimental Psychology: Human Perception \& Performance, 22, 869-878.

Koenderink, J. J., \& Lappin, J. S. (1996). Perturbation study of shading in pictures. Perception, 25, 1009-1026.

Koenderink, J. J., \& VAN Doorn, A. J. (1980). Photometric invariants related to solid shape. Optica Acta, 27, 981-996.

KoEnderink, J. J., \& VAN Doorn, A. J. (1983). Geometrical models as a general method to treat diffuse interreflections in radiometry. Journal of the Optical Society of America, 73, 843-850.

Koenderink, J. J., \& VAN Doorn, A. J. (1993). Illuminance critical points on generic smooth surfaces. Journal of the Optical Society of America A, 10, 844-854.

Koenderink, J. J., van Doorn, A. J., Christou, C., \& Lappin, J. S. (1996a). Perturbation study of shading in pictures. Perception, 25, 1009-1026.

Koenderink, J. J., van Doorn, A. J., Christou, C., \& Lappin, J. S. (1996b). Shape constancy in pictorial relief. Perception, 25, 155-164.

Koenderink, J. J., van Doorn, A. J., \& Kappers, A. M. L. (1992). Surface perception in pictures. Perception \& Psychophysics, 52, 487-496.

Koenderink, J. J., van Doorn, A. J., Kappers, A. M. L., \& Todd, J. T. (1997). The visual contour in depth. Perception \& Psychophysics, 59, 828-838.

Mamassian, P., Knill, D. C., \& Kersten, D. (1998). The perception of cast shadows. Trends in Cognitive Sciences, 2, 288-295.

Mingolla, E., \& Todd, J. T. (1986). Perception of solid shape from shading. Biological Cybernetics, 53, 137-151.

Norman, J. F., \& TodD, J. T. (1994). Perception of rigid motion in depth from optical deformations of shadows and occluding boundaries. Journal of Experimental Psychology: Human Perception \& Performance, 20, 343-356. 
Norman, J. F., Todd, J. T., \& Phillips, F. (1995). The perception of surface orientation from multiple sources of optical information. Perception \& Psychophysics, 57, 629-636.

Pentland, A. P. (1982). Finding the illuminant direction. Journal of the Optical Society of America, 72, 448-455.

Pentland, A. P. (1984). Local shading analysis. IEEE Transactions on Analysis \& Machine Intelligence, 6, 170-187.

Pentland, A. P. (1989). Shape information from shading: A theory about human perception. Spatial Vision, 4, 165-182.

Pollick, F. E., Watanabe, H., \& Kawato, M. (1996). Perception of local orientation from shaded images. Perception \& Psychophysics, 58, 762-780.

RAmachandran, V. S. (1988a). Perceiving shape from shading. Scientific American, 259, 76-83.

RAMACHANDRAN, V. S. (1988b). Perception of shape from shading. $\mathrm{Na}$ ture, 331, 163-166.

Reichel, F. D., \& TodD, J. T. (1990). Perceived depth inversion of smoothly curved surfaces due to image orientation. Journal of Experimental Psychology: Human Perception \& Performance, 16, 653-664.

Reichel, F. D., TodD, J. T., \& YILMAZ, E. (1995). Visual discrimination of local surface depth and orientation. Perception \& Psychophysics, 57, 1233-1240.

TodD, J. T. (1985). Perception of structure from motion: Is projective correspondence of moving elements a necessary condition? Journal of Experimental Psychology: Human Perception \& Performance, 11, 689-710.

Todd, J. T., Koenderink, J. J., van Doorn, A., \& Kappers, A. M. L. (1996). Effects of changing viewing conditions on the perceived structure of smoothly curved surfaces. Journal of Experimental Psychology: Human Perception \& Performance, 22, 695-706.

TodD, J. T., \& Mingolla, E. (1983). Perception of surface curvature and direction of illumination from patterns of shading. Journal of Experimental Psychology: Human Perception \& Performance, 9, 583-595.

Todd, J. T., \& Norman, F. J. (1999, November). Perceiving directions of illumination. Paper presented at the 40th Annual Meeting of the Psychonomic Society, Los Angeles.

TodD, J. T., \& ReIchel, F. D. (1989). Ordinal structure in the visual perception and cognition of smoothly curved surfaces. Psychological Review, 96, 643-657.

Troje, N. F., \& Siebeck, U. (1998). Illumination-induced apparent shift in orientation of human faces. Perception, 27, 671-680.

WINER, B. J. (1971). Statistical principles in experimental design (2nd ed.). New York: McGraw-Hill.

(Manuscript received September 20, 2000; accepted for publication July 2, 2001.) 